\title{
Exact results for a tunnel-coupled pair of trapped Bose-Einstein condensates
}

\author{
Huan-Qiang Zhout, Jon Links, Ross H. McKenzie, and Xi-Wen Guan† \\ Centre for Mathematical Physics, The University of Queensland, 4072, Australia \\ $\dagger$ Instituto de Física da UFRGS, Av. Bento Gonçalves 9500, Porto Alegre, RS - Brazil
}

\begin{abstract}
A model describing coherent quantum tunneling between two trapped Bose-Einstein condensates is shown to admit an exact solution. The spectrum is obtained by the algebraic Bethe ansatz. An asymptotic analysis of the Bethe ansatz equations leads us to explicit expressions for the energies of the ground and first excited states in the limit of weak tunneling and all energies for strong tunneling. The results are used to extract the asymptotic limits of the quantum fluctuations of the boson number difference between the two Bose-Einstein condensates and to characterize the degree of coherence in the system.
\end{abstract}

PACS numbers: 03.75.Fi, 05.30.Jp

Macroscopic quantum tunneling is one of the most fascinating phenomena in condensed matter physics [1,2]. Experimental realization of Bose-Einstein condensates (BEC's) in dilute atomic alkali gases has stimulated a diverse range of theoretical and experimental research activity [3 [7]. A particularly exciting possibility is that a pair of BEC's (such as a BEC trapped in a doublewell potential) may provide a model tunable system in which to observe macroscopic quantum tunneling. In this Letter we show that the canonical Hamiltonian for a pair of tunnel-coupled BEC's [5] has an exact solution. The model is also realizable in Josephson-coupled superconducting metallic nano-particles [8]. This connects the model to the rich and powerful mathematics associated with the algebraic Bethe ansatz [9] and provides a means to study the degradation of the coherence between the two BEC's which occurs as the tunnel coupling increases (or the size of the BEC decreases). The exactness of our approach means that it is not necessary to resort to approximations such as Gross-Pitaevskii mean-field theory and the phase-number formulation which become questionable in the coherent-incoherent crossover region.

The canonical Hamiltonian takes the form [5]

$$
\begin{aligned}
H= & \frac{K}{8}\left(N_{1}-N_{2}\right)^{2}-\frac{\Delta \mu}{2}\left(N_{1}-N_{2}\right) \\
& -\frac{\mathcal{E}_{\mathcal{J}}}{2}\left(a_{1}^{\dagger} a_{2}+a_{2}^{\dagger} a_{1}\right) .
\end{aligned}
$$

where $a_{1}^{\dagger}, a_{2}^{\dagger}$ denote the single-particle creation operators in the two wells and $N_{1}=a_{1}^{\dagger} a_{1}, N_{2}=a_{2}^{\dagger} a_{2}$ are the corresponding boson number operators. The total boson number $N_{1}+N_{2}$ is conserved and set to the fixed value of $N$. The physical meaning of the coupling parameters for different realizable systems may be found in Ref. [5]. It is useful to divide the parameter space into three regimes; viz. Rabi $\left(K / \mathcal{E}_{J}<<N^{-1}\right)$, Josephson $\left(N^{-1}<<K / \mathcal{E}_{J}<<N\right)$ and Fock $\left(N<<K / \mathcal{E}_{J}\right)$. In the Josephson region one expects coherent superposition of the two BEC's (Schrödinger cat states) to be possible whereas in the Fock region the two BEC's will be (in some sense) localised in the two separate wells. There is a correspondence between (11) and the motion of a pendulum [5]. In the Rabi and Josephson regimes this motion is semiclassical, unlike the case of the Fock regime. For both the Fock and Josephson regimes the analogy corresponds to a pendulum with fixed length, while in the Rabi regime the length varies. An important problem is to study the behaviour in the crossover regimes.

The exact solvability of (11) which we discuss here follows from the fact that it is mathematically equivalent to the discrete self-trapping dimer model studied by Enol'skii et al. [10], who solved the model through the algebraic Bethe ansatz. The Lax operator appearing in the Yang-Baxter equation which underlies the exact solution first appeared in 11]. The consequences of this result are legion. The exact solution gives a direct method, through numerical analysis, to investigate the nature of the system in the crossover regions between the Fock, Rabi and Josephson regimes. It also provides a means to test the limits of applicability of previous approximate treatments [12 18]. Solvability through the Yang-Baxter equation raises questions about the probability distribution of the energy level spacings 19 . Moreover, the formulation of the model through the algebraic version of the Bethe ansatz opens possibilities for the calculation of time-dependent form factors and correlation functions, as achieved in [20] for $\Delta \mu=0$.

In this Letter we will show that the exact solution allows for the analysis of the asymptotic behaviour of the energy spectrum in the limits of strong and weak tunneling. These results are used to extract the asymptotic limits of the quantum fluctuations of the relative particle number between the two BEC's and the degree of coherence in the system for the ground state. Our approach also makes it feasible to undertake an asymptotic analysis of the system at finite temperature which gives insight into the relative influence of thermal and quantum fluctuations (cf. [21]). We mention that these findings provide a useful tool in the numerical evaluation of 
the exact solution in the strong and weak tunneling regions. In contrast to most Bethe ansatz solvable models [9], the model discused here, as in the case of the AzbelHofstadter problem [22], is a quantum mechanical model rather than a quantum field theory; i.e., it has a fixed and finite number of degrees of freedom.

Bethe ansatz solution. Following the standard procedure of the algebraic Bethe ansatz [9], we can derive the Bethe ansatz equations (BAE). For $N$ total bosons the $\mathrm{BAE}$ for (1) can be written in the form

$$
\eta^{2}\left(v_{\alpha}^{2}-\omega^{2}\right)=\prod_{\beta \neq \alpha}^{N} \frac{v_{\alpha}-v_{\beta}-\eta}{v_{\alpha}-v_{\beta}+\eta}, \quad \alpha=1, \ldots, N .
$$

The parameters $\eta, \omega$ which naturally arise in the Bethe ansatz solution, along with a scaling factor $\kappa$, are related to the coupling constants of (11) through the identification

$$
K=2 \kappa \eta^{2}, \quad \Delta \mu=-2 \kappa \eta \omega, \quad \mathcal{E}_{\mathcal{J}}=2 \kappa .
$$

Each set of numbers $\left\{v_{\alpha}\right\}_{\alpha=1}^{N}$ which is a solution of the $\mathrm{BAE}$ defines an eigenstate of the Hamiltonian. For details of the derivation of (2) we refer to [10,20]. For such a solution, the eigenvector has the form

$$
\Psi\left(v_{1}, \ldots, v_{N}\right)=\prod_{\alpha=1}^{N} C\left(v_{\alpha}\right)|0\rangle
$$

with $C(u)=\left(u-\omega+\eta N_{2}\right) a_{1}^{\dagger}+\eta^{-1} a_{2}^{\dagger}$ and $|0\rangle$ the Fock vacuum.

For each $N$ we expect $N+1$ independent solutions of the BAE. Note that in the derivation of the BAE it is assumed that $v_{\alpha}$ are distinct for different $\alpha$. This is a result of the Pauli principle for Bethe ansatz solvable models as proved by Izergin and Korepin [23] for the one-dimensional $\delta$-function interaction boson gas. Their result may be adapted to the present case, which plays an important role in our asymptotic analysis of the Bethe ansatz solutions. From the BAE, we may derive the useful identity

$$
\prod_{\alpha=1}^{m} \eta^{2}\left(v_{\alpha}-\omega^{2}\right)=\prod_{\alpha=1}^{m} \prod_{\beta=m+1}^{N} \frac{v_{\alpha}-v_{\beta}-\eta}{v_{\alpha}-v_{\beta}+\eta}
$$

which will be used frequently.

For a given solution to the Bethe ansatz equations, the corresponding energy eigenvalue of the Hamiltonian is

$$
\begin{array}{r}
E=-\kappa\left(\eta^{-2} \prod_{\alpha}^{N}\left(1+\frac{\eta}{v_{\alpha}-u}\right)-\frac{\eta^{2} N^{2}}{4}-u \eta N-u^{2}\right. \\
\left.-\eta^{-2}+\omega^{2}+\left(u^{2}-\omega^{2}\right) \prod_{\alpha}^{N}\left(1-\frac{\eta}{v_{\alpha}-u}\right)\right) .
\end{array}
$$

Note that this expression is independent of the spectral parameter $u$ which can be chosen arbitrarily. The formula simplifies considerably with the choice $u=\omega$, by employing (2), which yields a polynomial form. However, for the purpose of an asymptotic analysis in the Rabi regime, it is more convenient to choose $u=0$, while for the Fock regime we use $u=\eta^{2}$.

Asymptotics. We start our analysis with the Rabi regime where $\eta^{2} N<<1$. From the BAE it is clear that $\eta^{2} v_{\alpha}^{2} \rightarrow 1$ as $\eta \rightarrow 0$, so that $v_{\alpha} \approx \pm \eta^{-1}$. However, when $\eta=0$ we know that the Hamiltonian is diagonalizable by using the Bogoliubov transformation, from which we can deduce that the solution of the BAE corresponding to the ground state must have $v_{\alpha} \approx \eta^{-1}$. Excitations correspond to changing the signs of the leading terms in the Bethe ansatz roots. To study the asymptotic behaviour for the $m$ th excited state, we set

$$
\begin{aligned}
& v_{\alpha} \approx-\eta^{-1}+\epsilon_{\alpha}+\eta \delta_{\alpha}, \quad \alpha=1, \ldots, m, \\
& v_{\alpha} \approx \eta^{-1}+\epsilon_{\alpha}+\eta \delta_{\alpha}, \quad \alpha=m+1, \ldots, N,
\end{aligned}
$$

with the convention that the ground state corresponds to $m=0$.

From the leading terms of the BAE for $v_{\alpha}, \alpha \leq m$ we find

$$
\epsilon_{\alpha}=\sum_{\beta \neq \alpha}^{m} \frac{1}{\epsilon_{\alpha}-\epsilon_{\beta}}
$$

which implies

$$
\sum_{\alpha=1}^{m} \epsilon_{\alpha}=0, \quad \sum_{\alpha=1}^{m} \epsilon_{\alpha}^{2}=\frac{m(m-1)}{2} .
$$

In a similar fashion we have for $m<\alpha \leq N$

$$
\epsilon_{\alpha}=-\sum_{\alpha \neq \beta=m+1}^{N} \frac{1}{\epsilon_{\alpha}-\epsilon_{\beta}},
$$

which implies

$$
\sum_{\alpha=m+1}^{N} \epsilon_{\alpha}=0, \quad \sum_{\alpha=m+1}^{N} \epsilon_{\alpha}^{2}=-\frac{(N-m)(N-m-1)}{2} .
$$

It is clear from (6) and (6) why the Pauli exclusion principle applies in the present case. In the asymptotic expansion for $v_{\alpha}, \epsilon_{\alpha}$ is assumed finite. However, if $v_{\alpha}=v_{\beta}$ for some $\alpha, \beta$, then $\epsilon_{\alpha}=\epsilon_{\beta}$ and (5) and (6) imply that $\epsilon_{\alpha}, \epsilon_{\beta}$ are infinite which is a contradiction. Hence $v_{\alpha}$ must be distinct for different $\alpha$. Note also that for this approximation to be valid we require $\eta^{-1}>>\epsilon_{\alpha}$. However, we see that $\left|\epsilon_{\alpha}\right|$ is of the order of $N^{1 / 2}$. Thus our approximation will be valid for $\eta N^{1 / 2}<<1$, which is precisely the criterion for the Rabi region and consequently $N$ cannot be arbitrarily large for fixed $\eta$, or vice versa.

Now we go to the next order. From (2) we find 


$$
\begin{gathered}
\sum_{\alpha=1}^{m} \delta_{\alpha}=-\frac{m(m-1)}{4}+\frac{m(m-N)}{2}-\frac{m \omega^{2}}{2} \\
\sum_{\alpha=m+1}^{N} \delta_{\alpha}=-\frac{(N-m)(N-m-1)}{4}+\frac{m(m-N)}{2} \\
+\frac{(N-m) \omega^{2}}{2}
\end{gathered}
$$

which using (3) leads us to the result

$$
\frac{E_{m}}{\kappa} \approx-N+2 m-\frac{\eta^{2} \omega^{2}(N-2 m)}{2}+\frac{\eta^{2} N}{4}+\frac{\eta^{2}}{2} m(N-m) .
$$

The energy level spacings $\Delta_{m}=E_{m}-E_{m-1}$ are thus

$$
\Delta_{m} \approx \kappa\left(2+\eta^{2} \omega^{2}+\frac{\eta^{2}}{2}(N-2 m+1)\right) .
$$

One may check that $\Delta_{m} / N$ is of the order of $N^{-1}$. This indicates that the Rabi regime is semiclassical [5]. Our value for the gap between the ground and first excited state agrees, to leading order in $\eta^{2} N$, with the GrossPitaevskii mean-field theory [13] which gives a Josephson plasma frequency of $\omega_{J}=2 \kappa\left(1+\eta^{2} N / 2\right)^{1 / 2}$.

Now we look at the asymptotic behaviour of the BAE in the Fock regime $\eta^{2}>>N$. It is necessary to distinguish the following cases: (i) $\omega=0$ and (ii) $\omega \neq 0$.

(i) $\omega=0$. In this case, it is appropriate to consider the permutation operator $P$ which interchanges the labels 1 and 2 in (1). For $\omega=0, P$ commutes with the Hamiltonian, and any eigenvector of the Hamiltonian is also an eigenvector of $P$ with eigenvalue \pm 1 . Therefore the Hilbert space splits into the direct sum of two subspaces corresponding to the symmetric and antisymmetric eigenfunctions. From now on we restrict ourselves to the case when $N$ is even, i.e., $N=2 M$, although a similar calculation is also applicable to the case when $N$ is odd. A careful analysis leads us to conclude that the ground state lies in the symmetric subspace. The asymptotic form of the roots of the BAEs for the ground state takes the "string"-like structure

$$
\begin{aligned}
v_{j \pm} \approx-(M & -j) \eta \pm i \frac{C_{M}^{j}}{(j-1) !} \eta^{-(2 j-1)} \\
& +M(M+1) \eta^{-3} \delta_{j 1}, \quad j=1, \cdots, M .
\end{aligned}
$$

where $C_{M}^{j}$ is a binomial coefficient. For this asymptotic ansatz to be valid, we require that any term in the asymptotic expansion should be much smaller than those preceeding. This yields $\eta^{2}>>N$ which coincides with the defining condition for the Fock region. Throughout, the Pauli exclusion principle has been taken into account to exclude any possible spurious solutions of the BAE.

The above structure clearly indicates that in the ground state the $N$ bosons fuse into $M$ "bound" states and excitations correspond to a breakdown of these bound states. Specifically, the first and second excited states correspond to the breakdown of the bound state at $-(M-1) \eta$, with the first excited state in the antisymmetric subspace and the second excited state in the symmetric subspace. Explicitly, we can write down the spectral parameter configurations for the first two excited states

$$
\begin{aligned}
& v_{1+} \approx-M \eta+a_{1+} \eta^{-3}, \quad v_{1-} \approx-(M-1) \eta+a_{1-} \eta^{-3}, \\
& v_{j \pm} \approx-(M-j) \eta+a_{j \pm} \eta^{-(2 j-1)}, \quad j=2, \cdots, M,
\end{aligned}
$$

with

$$
\begin{aligned}
& a_{1+} \approx-\frac{M+1}{2}, \quad a_{1-} \approx \frac{M(M+1)}{2}, \\
& a_{2 \pm} \approx \frac{-(M-1)^{2} \pm(M-1) \sqrt{13 M^{2}+10 M+1}}{12}, \\
& a_{3 \pm} \approx \pm \frac{(M-1)(M-2) \sqrt{2 M(M+1)}}{24}, \\
& a_{j \pm} \approx \frac{M-j+1}{\sqrt{(j+1) j(j-1)(j-2)}} a_{j-1, \pm}, \quad j=3, \cdots, M,
\end{aligned}
$$

for the (antisymmetric) first excited state and

$$
\begin{aligned}
& a_{1+} \approx-\frac{(M+1)(2 M+1)}{2}, \quad a_{1-} \approx-\frac{M(M+1)}{2}, \\
& a_{2 \pm} \approx \frac{-(M-1)^{2} \pm i(M-1) \sqrt{11 M^{2}+14 M-1}}{12}, \\
& a_{3 \pm} \approx \pm i \frac{(M-1)(M-2) \sqrt{2 M(M+1)}}{24}, \\
& a_{j \pm} \approx \frac{M-j+1}{\sqrt{(j+1) j(j-1)(j-2)}} a_{j-1, \pm}, \quad j=3, \cdots, M,
\end{aligned}
$$

for the (symmetric) second excited state. The breakdown of the bound state at $-(M-j) \eta, j=2, \cdots, M$ results in the higher excited states.

Substituting these results into (3) leads us to the asymptotic ground state energy

$$
E_{0} \approx-2 \kappa \eta^{-2} M(M+1),
$$

while for the first and second excited states we have

$$
\begin{aligned}
& E_{1} \approx \kappa \eta^{2}-\kappa \eta^{-2} \frac{M^{2}+M-2}{3}, \\
& E_{2} \approx \kappa \eta^{2}+\kappa \eta^{-2} \frac{5 M^{2}+5 M+2}{3} .
\end{aligned}
$$

In contrast to the Rabi regime, the Fock regime is not semiclassical, as the ratio of the gap $\Delta$ and $N$ is of finite order when $N$ is large.

We can perform a similar analysis for odd $N$. In this case, the gap between the ground and the first excited states is proportional to $\kappa \eta^{-2}$ instead of $\kappa \eta^{2}$. This indicates there is a strong parity effect in the Fock regime. Its possible physical implication remains to be explored. 
(ii) $\omega \neq 0$. In this case the root structure is somewhat more complicated than for $\omega=0$, so we will not present the details. We remark however that our calculations show that up to order $\eta^{-2}$ the ground state energy eigenvalue takes the same form as in the case $\omega=0$. Actually, the leading contribution arising from the $\omega$ term appears only as $\omega^{2} \eta^{-4}$. This means that the results presented below are applicable for all values of $\omega$ (or equivalently $\Delta \mu)$.

Quantum fluctuations and coherence factor. Although it is difficult to define rigorously [5], the relative phase between Bose-Einstein condensates has been useful in understanding interference experiments [6,7,24]. By definition, the relative phase $\Phi$ is conjugate to the relative number of atoms in the two condensates $n \equiv N_{1}-N_{2}$. Using the Feynmann-Hellman theorem, we find that

$$
<\Delta n^{2}>=8 \frac{\partial E}{\partial K}-4\left(\frac{\partial E}{\partial \Delta \mu}\right)^{2} .
$$

For the ground state in the limit of strong tunneling (i.e., Rabi regime), $\left\langle\Delta n^{2}\right\rangle=N-\left(\Delta \mu N / \mathcal{E}_{J}\right)^{2}$. In the case of weak tunneling (i.e., Fock regime), $\left\langle\Delta n^{2}\right\rangle \approx$ $2 N(N+2)\left(\mathcal{E}_{J} / K\right)^{2}$. The degree of coherence between the two BEC's can be discussed in terms of [5]

$$
\alpha \equiv \frac{1}{2 N}<a_{1}^{\dagger} a_{2}+a_{2}^{\dagger} a_{1}>=-\frac{1}{N} \frac{\partial E}{\partial \mathcal{E}_{J}}
$$

where the last identity follows from the FeynmannHellmann theorem. In terms of the relative phase $\Phi$, $\alpha \doteq<\cos \Phi>$ 21]. In the strong coupling limit, $\alpha \approx 1-N^{-1}(\Delta \mu)^{2} /\left(8 \mathcal{E}_{J}\right)^{2}$, indicating very close to full coherence in the ground state. In the opposite limit, we have $\alpha \approx 2(N+2) \mathcal{E}_{J} / K<<1$, indicating the absence of coherence.

The above results give the first order corrections to the results presented in 6621] for the number fluctuations and the coherence factor at zero temperature. Moreover, we can extend this approach to gain insight into the effects of thermal fluctuations. This problem was recently studied in 21] using Gross-Pitaevskii theory. In the Fock regime, the energy gap is large, and so for the low temperature thermodynamics it is sufficient to consider the first two excited states, for which the calculations are straightforward. However, in the Rabi regime the small energy scale requires us to consider the entire spectrum. The asymptotic form of the partition function is found to be (valid for all temperatures)

$$
\begin{aligned}
Z & =\sum_{m} \exp \left(-E_{m} / T\right) \\
& \approx \frac{\sinh \frac{\beta}{2}(N+1)}{\sinh \frac{\beta}{2}}+\frac{\beta K}{4 \mathcal{E}_{J}} \exp (\beta N) \hat{\mathcal{D}}\left(\frac{1-\exp \beta(N+1)}{1-\exp \beta}\right),
\end{aligned}
$$

with $\beta=-\frac{2 \kappa}{T}$ (we set Boltzmann's constant $k_{B}=1$ ) and $\hat{\mathcal{D}}$ is the differential operator

$$
\hat{\mathcal{D}} \equiv-\frac{(\Delta \mu)^{2} N}{4 \mathcal{E}_{J} K}+\frac{N}{4}+\left(\frac{(\Delta \mu)^{2}}{2 \mathcal{E}_{J} K}+\frac{N}{2}\right) \frac{\partial}{\partial \beta}-\frac{1}{2} \frac{\partial^{2}}{\partial^{2} \beta} .
$$

In the usual way it is possible to derive a number of physical quantities from $Z$. For example, the temperature dependent coherence factor is simply

$$
\alpha(T)=\frac{T}{N} \frac{\partial \ln Z}{\partial \mathcal{E}_{J}}
$$

and an expression for the particle number fluctuations can be found in a similar manner. This provides an analytic means to investigate the thermal effects for the Rabi regime. In order to investigate the crossover to weaker tunneling, it is possible to solve the BAE numerically to compute these quantities. This will be the subject of future work.

In conclusion, we have shown that the canonical Hamiltonian describing quantum tunneling between a pair of BEC's is integrable in the sense of the algebraic Bethe ansatz. An closed form expression was given for all of the energy eigenstates and eigenvalues in terms of the solutions of the Bethe ansatz equations. Our approach makes the calculation of form factors [20] and correlation functions possible without any of the limitations associated with Gross-Pitaevskii mean-field theory or the number-phase representation.

We thank J. Corney, G.J. Milburn, and M.D. Gould for helpful discussions. This work was supported by the Australian Research Council.

* E-mail: hqz@maths.uq.edu.au

[1] J.R. Friedman et al., Nature 406, 43 (2000).

[2] C.H. van der Wal et al., Science 290, 773 (2000).

[3] A.S. Parkin and D.F. Walls, Phys. Rep. 303, 1 (1998).

[4] F. Dalfovo, S. Giorgini, L.P. Pitaevskii and S. Stringari, Rev. Mod. Phys. 71, 463 (1999).

[5] A.J. Leggett, Rev. Mod. Phys. 73, 307 (2001).

[6] C. Orzel et al., Science 291, 2386 (2001).

[7] F.S. Cataliotti et al., Science 293, 843 (2001).

[8] Y. Makhlin, G. Schön, and A. Shnirman, Rev. Mod. Phys. 73, 357 (2001).

[9] V. E. Korepin, N. M. Bogoliubov, and G. Izergin Quantum inverse scattering method and correlation functions (Cambridge University Press, 1993, Cambridge).

[10] V.Z. Enol'skii, M. Salerno, N.A. Kostov and A.C. Scott, Phys. Scr. 43, 229 (1991).

[11] V.B. Kuznetsov and A.V. Tsiganov, J. Phys. A22, L73 (1989).

[12] G.J. Milburn, J. Corney, E.M. Wright, and D.F. Walls, Phys. Rev. A55, 4318 (1997).

[13] A. Smerzi, S. Fantoni, S. Giovanazzi and S.R. Shenoy, Phys. Rev. Lett. 79, 4950 (1997); G.-S. Paroanu, S. Kohler, F. Sols and A.J. Leggett, cond-mat/0108551. 
[14] I. Zapata, F. Sols and A.J. Leggett, Phys. Rev. A57, R28 (1998).

[15] S. Raghavan, A. Smerzi, S. Fantoni and S.R. Shenoy, Phys. Rev. A59, 620 (1999).

[16] J. Javanainen and M. Yu. Ivanov, Phys. Rev. A60, 2351 (1999).

[17] I. Marino, S. Raghavan, S. Fantoni, S.R. Shenoy and A. Smerzi, Phys. Rev. A60, 487 (1999).

[18] J.R. Anglin, P. Drummond and A. Smerzi, Phys Rev. A64, 063605 (2001).

[19] D. Poilblanc, T. Ziman, J. Bellisard, F. Mila, and G. Montambaux, Europhys. Lett. 22, 537 (1993); T.C. Hsu and J.C.A. d'Auriac, Phys. Rev. B47, 14291 (1993); H.
Meyer, J.C.A. d'Auriac, J.M. Maillard, Phys. Rev. E55, 5380 (1997).

[20] J. Links and H.-Q. Zhou, nlin.SI/0202041.

[21] L. Pitaevskii and S. Stringari, Phys. Rev. Lett. 87, 180402 (2001).

[22] P.B. Wiegmann and A. Zabrodin, Phys. Rev. Lett. 72, 1890 (1994); A.G. Abanov, J.C. Talstra and P.B. Wiegmann, Phys. Rev. Lett. 81, 2112 (1998).

[23] A.G. Izergin and V.E. Korepin, Lett. Math. Phys. 6 (1982) 283.

[24] D.S. Hall, M.R. Mathews, C.E. Wieman and E.A. Cornell, Phys. Rev. Lett. 81, 1539 (1998). 